\title{
Clinical mid-term outcomes of the Chinese-made CL-V bileaflet mechanical heart valve in Chinese patients
}

\author{
Liang $\mathrm{Qi}^{1,2 \#}$, Shidong Liu ${ }^{1,2 \#}$, Yan Qiang ${ }^{3,4}$, Honglin Zhao ${ }^{1}$, Ruisheng Liu ${ }^{1}$, Dianwei Cheng ${ }^{1}$, Bing Song ${ }^{1}$, \\ Quanlin Guan ${ }^{2}$
}

${ }^{1}$ Department of Cardiovascular Surgery, The First Hospital of Lanzhou University, Lanzhou, China; ${ }^{2}$ The First School of Clinical Medicine, Lanzhou University, Lanzhou, China; ${ }^{3}$ Energy and Power Engineering College, Lanzhou University of Technology, Lanzhou, China; ${ }^{4}$ School of Energy and Environment, Southeast University, Nanjing, China

Contributions: (I) Conception and design: L Qi, R Liu; (II) Administrative support: B Song, Q Guan; (III) Provision of study materials or patients: H Zhao, D Cheng; (IV) Collection and assembly of data: L Qi, S Liu; (V) Data analysis and interpretation: S Liu, Y Qiang; (VI) Manuscript writing: All authors; (VII) Final approval of manuscript: All authors.

"These authors contributed equally to this work.

Correspondence to: Bing Song. Department of Cardiovascular surgery, The First Hospital of Lanzhou University, Lanzhou 730000, China. Email: songbinldyy@163.com; Quanlin Guan. Surgical oncology, The First Hospital of Lanzhou University, Lanzhou 730000, China. Email: 18368914891@163.com.

Background: The CL-V full-carbon bileaflet mechanical heart valve is a novel Chinese-made prosthetic valve. This study evaluated the mid-term outcomes of the CL-V bileaflet mechanical heart valve after implantation in Chinese patients.

Methods: This study retrospectively enrolled a total of 38 consecutive patients who underwent elective mechanical heart valve replacement (MHVR) with two different valve types from April 2004 and May 2010, including 18 patients with the CL-V bileaflet mechanical heart valve (44.4\% male, mean age $47.4 \pm 6.2$ years, mean body weight $64.7 \pm 11.9 \mathrm{~kg})$ and 20 patients with the St. Jude mechanical heart valve $(45.0 \%$ male, mean age $49.7 \pm 7.6$ years, mean body weight $66.1 \pm 11.1 \mathrm{~kg}$ ). All patients underwent follow-up clinical evaluations in the outpatient department at all-time points.

Results: No complications occurred during the mean 61.3 months follow-up time (range, 47102 months). The cardiothoracic ratios $(52.7 \% \pm 4.5 \%$ vs. $50.1 \% \pm 4.0 \%)$, left atrium diameter $(46.5 \pm 7.6$ vs. $44.8 \pm 9.3 \mathrm{~mm})$, left ventricular diastolic diameter $(47.6 \pm 4.9$ vs. $48.2 \pm 8.5 \mathrm{~mm})$ and left ventricular ejection fraction $(65.4 \% \pm 8.7 \%$ vs. $64.5 \% \pm 8.0 \%)$ were not significantly different between the two groups $(\mathrm{P}>0.05)$. Transthoracic Doppler echocardiography showed that the hemodynamic indexes were not significantly different between the two groups at 1 year and 3 years $(\mathrm{P}>0.05)$. Furthermore, no significant differences were found between the two groups in hemocompatibility indexes at both 6 months and 3 years postoperatively $(\mathrm{P}>0.05)$.

Conclusions: The mid-term follow-up results of the CL-V bileaflet mechanical heart valve were similar to those of the St. Jude Medical heart valve, which showed stable hemodynamics and good blood compatibility. Chinese-made CL-V bileaflet mechanical heart valves can be a substitute for St. Jude Medical heart valves, and can be widely used in cardiac surgery.

Trial registration: Chinese Clinical Trial Registry ChiCTR2000034158.

Keywords: Heart valve disease; CL-V bileaflet mechanical heart valve; St. Jude Medical heart valve

Submitted Jul 08, 2020. Accepted for publication Oct 24, 2020.

doi: $10.21037 /$ jtd-20-2644

View this article at: http://dx.doi.org/10.21037/jtd-20-2644

(c) Journal of Thoracic Disease. All rights reserved. 


\section{Introduction}

Valvular heart disease (VHD) is a growing public health problem (1). Congenital and acquired VHD remains a predictable cause of heart failure and an important cause of sudden death $(2,3)$. The prevalence of VHD in industrialized countries is estimated to be $2.5 \%$, and increases with age (3). After the age of 65, the incidence of VHD increases significantly (4). Of all VHD subtypes, aortic valve disease has the worst prognosis and a higher mortality rate (5).

The CL-V bileaflet is a novel domestic mechanical valve prosthesis, consisting of a valve leaflet, valve frame and suture ring (6). The valve leaflet is coated with pyrocarbon and tungsten-containing graphite impervious to $\mathrm{X}$-rays, with a convex pivot structure on both sides of the valve leaflet and ' 8 ' shaped grooves in the valve frame which constitute a movable hinge joint. The opening angle of the valve leaflet is $82^{\circ}$ and the closing angle is $25^{\circ}$. The valve frame is made of pure pyrolytic carbon. The material for the rotatable suture ring is made of polyester fabric for medical use. The St. Jude bileaflet is produced by St. Jude Medical company of the United States, and the valve frame and leaflet are made of graphite coated with pyrolytic carbon. Two semicircular valve leaflets control the opening and closing with an opening angle of $85^{\circ}$, and the suture ring is made of polyester fabric for medical use.

The bileaflets which have achieved long-term clinical application, especially the St. Jude and Carbomedics bileaflets, constitute more than 70 percent of clinical valve types and are recognized as the most excellent artificial mechanical heart valves (7-9). Most previous studies are case report, or only reported short-term clinical effects. The CL-V bileaflet is a brand new Chinese-made prosthetic valve. The present study is the first trial evaluating the midterm outcome results of the CL-V bileaflet mechanical heart valve after implantation in Chinese patients. We present the following article in accordance with the STROBE reporting checklist (available at http://dx.doi.org/10.21037/jtd-202644).

\section{Methods}

\section{Study patients}

The present study retrospectively enrolled a total of 38 consecutive patients who underwent mechanical heart valve replacement (MHVR) with two different valve types in the First Hospital of Lanzhou University from April
2004 and May 2010. Patients were eligible for inclusion if they met all of the following criteria: (I) patients aged $\geq 18$ years old; (II) patients diagnosed on the basis of medical history, physical examination, electrocardiogram, chest $\mathrm{X}$-ray and transthoracic Doppler echocardiography; (III) patients underwent MHVR surgery with a Chinese-made CL-V bileaflet or St. Jude mechanical heart valve (St. Jude Medical, Inc., St. Paul, MN, USA). Patients were excluded if they met any of the following criteria: (I) patients aged $\geq 50$ years old; (II) coronary heart disease; (III) pregnancy; (IV) dialysis; (V) aortic dissection; (VI) critical perioperative state; (VII) recent neurologic event before operation; (VIII) severe renal insufficiency (serum creatinine $>150 \mu \mathrm{mol} / \mathrm{L}$ ) before operation; (IX) bioprosthetic heart valve replacement; $(\mathrm{X})$ intra-aortic balloon counter pulsation. Informed consent was obtained from all individual participants included in the study. The study was approved by the ethics committee of the First Hospital of Lanzhou University (LDYYLL2019-230), and the study was registered with the Chinese Clinical Trial Registry, registration number ChiCTR2000034158. All procedures performed in this study involving human participants were in accordance with the Declaration of Helsinki (as revised in 2013).

\section{Surgical techniques and postoperative anticoagulation}

All patients underwent a median sternotomy incision at medium temperature ranging from 27 to $32{ }^{\circ} \mathrm{C}$ under general anesthesia with cardiac-pulmonary bypass. The operation was performed via a membrane oxygenator under cardiac arrest and the patients were perfused with cold cardioplegia solution. Continuous suture was performed for mitral valve replacement, and interrupted suture was performed for aortic valve replacement. If a left atrial thrombus was found, left atrial embolectomy was performed. If tricuspid insufficiency was severe, valvuloplasty was not performed. After the operation, assisted circulation was provided to patients until circulation, breathing, arterial blood gas and electrolyte levels were satisfactory. The respirator was then stopped, the respiratory tube was drawn, protamine was used for neutralization, then hemostasis and sternal closure were performed. After the patient was transferred to the ICU, a respirator was used continuously to control breathing, and other treatments such as electrocardiograph monitoring and invasive blood pressure monitoring were implemented.

After the patient was stabilized, the patient was 
transferred to a general ward. Warfarin (domestically produced) was taken orally at 18:00 on the $2 \mathrm{nd}$ or $3 \mathrm{rd}$ day postoperatively with a first dose of 3.75 to $5 \mathrm{mg}$. Anticoagulant detection was conducted 48 hours later, then the dose was adjusted according to the monitoring data. The prothrombin time (PT) was adjusted to 1.5 times that of the control value, and the international normalized ratio (INR) was 1.5 to 2.5. After the operation, no patient took anti-platelet drugs.

\section{Follow-up and study endpoints}

All patients returned to the outpatient department for reexamination at 1, 3, 6 and 12 months after discharge and annually thereafter. Examination included: inquiry about subjective symptoms, physical examination, laboratory examinations, chest X-ray, transthoracic Doppler echocardiography, evaluation of cardiac function, and observations of prosthetic valve-related complications. The details of the follow-up were recorded on a form and follow-up examination data were filed.

The primary endpoint of the present study was a composite of complications such as death, stroke, transient ischemic attack (TIA), myocardial infarction, thromboembolism, life-threatening or disabling bleeding, acute kidney injury and residual shunt. The secondary endpoints of the present study included: (I) cardiac function indexes; (II) hemodynamic indexes and (III) hemocompatibility indexes.

\section{Statistical analysis}

Continuous variables are expressed as mean \pm standard deviation (SD) and categorical variables are expressed as numbers (percentages). The statistical significance of continuous data were tested using a Student's $t$ test or the Mann-Whitney $\mathrm{U}$ test, depending on whether there was a Gaussian distribution. A Chi-square test or Fisher's exact test was used to determine statistical significance of categorical data. A P value of less than 0.05 was considered to be statistically different. All data were calculated and analyzed using Software Package for Statistics and Simulation (SPSS; version 22.0, IBM Corp Armonk, NY, USA).

\section{Results}

\section{Patient demographics and baseline characteristics}

A total 38 patients met the inclusion criteria and underwent
MHVR with the CL-V bileaflet or the St. Jude mechanical heart valve. According to the type of valve, patients enrolled in the study were divided into two groups: the CL-V group, including 18 patients with the CL-V bileaflet mechanical heart valve $(44.4 \%$ male, mean age $47.4 \pm 6.2$ years, mean body weight $64.7 \pm 11.9 \mathrm{~kg}$ ), and the SJM group including 20 patients with the St. Jude mechanical heart valve (45.0\% male, mean age $49.7 \pm 7.6$ years, mean body weight $66.1 \pm 11.1 \mathrm{~kg}$ ). A total of $20 \mathrm{CL}-\mathrm{V}$ bileaflets were implanted including 18 valves in the mitral position (including $125 \mathrm{~mm}$ valve and $1727 \mathrm{~mm}$ valves) and 2 valves in the aortic position (2 $23 \mathrm{~mm}$ valves). Mitral valve replacement was performed in 16 patients and combined aortic and mitral valve replacement was performed in 2 patients. A total of 22 St. Jude Medical heart valves were implanted, including 19 valves in the mitral position (including $125 \mathrm{~mm}$ valve and $1827 \mathrm{~mm}$ valves) and 3 valves in the aortic position (3 $23 \mathrm{~mm}$ valves). The patient demographics and baseline characteristics are shown in Table 1, and the two groups were well balanced in regards to these baseline factors. All patients returned to the outpatient department for reexamination at 1, 3, 6 and 12 months after discharge and annually thereafter.

\section{Primary endpoints}

At mean 61.3 months follow-up time (range, 47-102 months), there were no complications such as death, stroke, TIA, myocardial infarction, thromboembolism, lifethreatening or disabling bleeding, acute kidney injury or residual shunt.

\section{Secondary endpoints}

\section{Cardiac function indexes}

A comparison of cardiac function indexes between the CL-V and SJM groups is shown in Table 2. Cardiothoracic ratio $(\mathrm{C} / \mathrm{T})$, left atrium diameter (LAD), left ventricular diastolic diameter (LVDD), left ventricular ejection fraction (LVEF), and left ventricular fractional shortening (LVFS) were not statistically different at 1 year and 3 years follow-up.

NYHA symptoms improved at similar rates in both groups as shown in Figure 1. NYHA class 1 had 0, 5, 10 cases in CL-V group, and 0, 3, 11 cases in SJM group, NYHA class 2 had 6, 8, 6 cases in CL-V group, and 5, 9, 5 cases in SJM group, NYHA class 3 had 7, 3, 2 cases in CL-V group, and 8, 5, 3 cases in SJM group, NYHA class 4 had 5, 2, 0 cases in CL-V group, and 7, 3, 1 cases in SJM 
Table 1 Baseline characteristics of patients

\begin{tabular}{|c|c|c|c|}
\hline Characteristics & CL-V group $(n=18)$ & SJM group (n=20) & $P$ value \\
\hline Male, n (\%) & $11(61.1)$ & $9(45.0)$ & 0.97 \\
\hline Weight, kg & $64.7 \pm 11.9$ & $66.1 \pm 11.1$ & 0.62 \\
\hline NYHA ${ }^{a}$ class III or IV, n (\%) & $12(66.7)$ & $15(75.0)$ & 0.57 \\
\hline Diabetes, n (\%) & $3(16.7)$ & $2(10.0)$ & 0.90 \\
\hline Atrial fibrillation, $\mathrm{n}(\%)^{\mathrm{b}}$ & $6(33.3)$ & $4(20.0)$ & 0.57 \\
\hline Arial septal defect, $\mathrm{n}(\%)$ & $1(5.6)$ & $0(0.0)$ & 0.96 \\
\hline Patent ductus arteriosus, n (\%) & $1(5.6)$ & $0(0.0)$ & 0.96 \\
\hline Left atrial thrombus, n (\%) & $2(11.1)$ & $3(15.0)$ & 1.00 \\
\hline \multicolumn{4}{|l|}{ Operative characteristics, n (\%) } \\
\hline $\operatorname{AVR}^{\mathrm{c}}$ & $0(0.0)$ & $1(5.0)$ & 1.00 \\
\hline $\mathrm{MVR}^{\mathrm{d}}$ & $16(88.9)$ & 17 (85.0) & 1.00 \\
\hline $\mathrm{BVR}^{\mathrm{e}}$ & $2(11.1)$ & $2(10.0)$ & 1.00 \\
\hline \multicolumn{4}{|c|}{ Surgical valve labeled size $(\mathrm{mm}), \mathrm{n}(\%)$} \\
\hline 23 & $2(10.0)$ & $3(13.6)$ & 1.00 \\
\hline 25 & $1(4.8)$ & $1(4.3)$ & 1.00 \\
\hline
\end{tabular}

Continuous variables are expressed as mean \pm SD; categorical variables are expressed as number (percentage). ${ }^{\text {, }}$, New York Heart Association functional class; ${ }^{b}$, includes transient, persistent or permanent atrial fibrillation; ${ }^{c}$, aortic valve replacement; ${ }^{d}$, mitral valve replacement; ${ }^{e}$, bivalve replacement. SJM, St. Jude mechanical heart valve.

Table 2 Comparison of cardiac function indexes at 1 year and 3 years

\begin{tabular}{|c|c|c|c|c|c|c|}
\hline Indexes & \multicolumn{3}{|c|}{1 year } & \multicolumn{3}{|c|}{3 years } \\
\hline $\mathrm{C} / \mathrm{T}(\%)$ & $58.4 \pm 4.7$ & $56.9 \pm 5.3$ & 0.34 & $52.7 \pm 4.5$ & 50.14 .0 & 0.95 \\
\hline LAD (mm) & $48.5 \pm 3.4$ & $47.6 \pm 3.3$ & 0.39 & $46.5 \pm 7.6$ & 44.89 .3 & 0.87 \\
\hline LVDd (mm) & $50.1 \pm 2.6$ & $51.5 \pm 3.2$ & 0.13 & $47.6 \pm 4.9$ & 48.28 .5 & 0.97 \\
\hline LVFS (\%) & $35.1 \pm 1.2$ & $35.8 \pm 2.2$ & 0.25 & $35.6 \pm 7.6$ & $34.4 \pm 4.9$ & 0.98 \\
\hline
\end{tabular}

SJM, St. Jude mechanical heart valve; C/T, cardiothoracic ratio; LAD, left atrium diameter; LVDD, left ventricular diastolic diameter; LVEF, left ventricular ejection fraction; LVFS, left ventricular fractional shortening. 


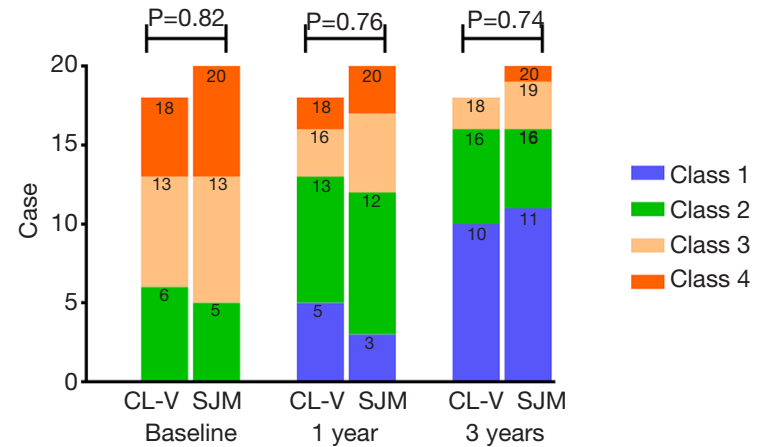

Figure 1 NYHA functional class at baseline, 1 year and 3 years. Based relevant symptoms assessing patients' NYHA Function grade, no difference between the CL-V group and SJM group at all-time points up to 3 years post-surgery. NYHA, New York Heart Association. SJM, St. Jude mechanical heart valve.

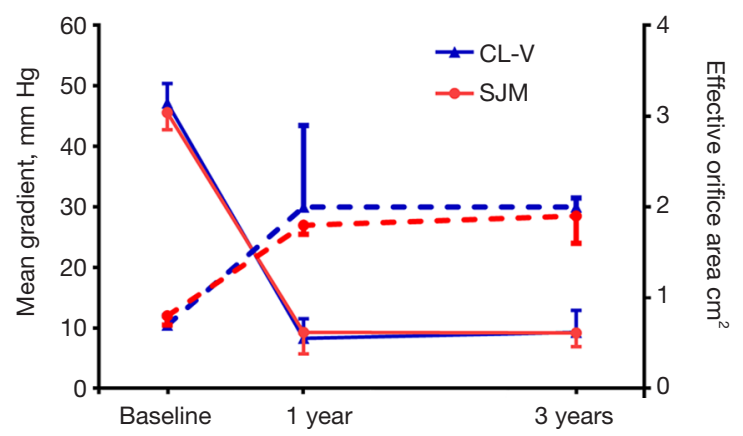

Figure 2 Aortic valve mean gradient and effective orifice area. Based transthoracic Doppler echocardiography assessing mean gradients and effective orifice areas of aortic valve, no difference between the CL-V group and SJM group at all-time points up to 3 years post-surgery. SJM, St. Jude mechanical heart valve.

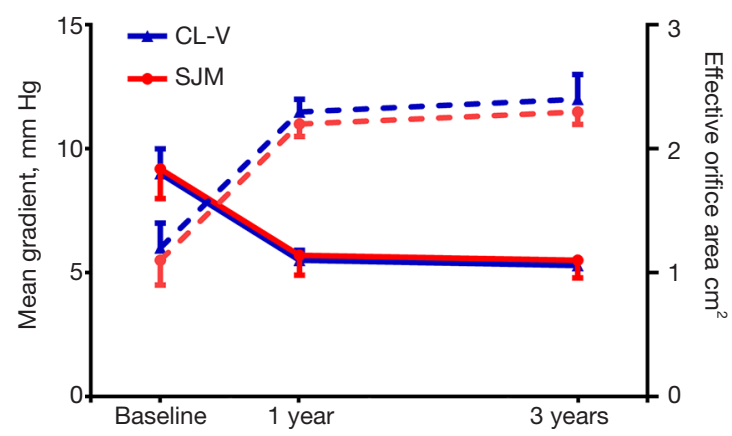

Figure 3 Mitral valve mean gradient and effective orifice area. Based transthoracic Doppler echocardiography assessing mean gradients and effective orifice areas of mitral valve, no difference between the CL-V group and SJM group at all-time points up to 3 years post-surgery. SJM, St. Jude mechanical heart valve. group at baseline, 1 year and 3 year respectively.

\section{Hemodynamic indexes}

For the aortic valve, the mean gradients at baseline $(47.2 \pm 3.2$ vs. $45.6 \pm 2.8 ; \mathrm{P}=0.59)$, 1 year $(8.3 \pm 3.3$ vs. $9.3 \pm 3.6$; $\mathrm{P}=0.45)$, and 3 years $(8.5 \pm 1.2$ vs. $9.2 \pm 1.1 ; \mathrm{P}=0.50)$ were not significantly different. Furthermore, effective orifice areas at baseline $(0.7 \pm 0.1$ vs. $0.8 \pm 0.1 ; \mathrm{P}=0.41), 1$ year $(2.0 \pm 0.9$ vs. $1.8 \pm 0.1 ; \mathrm{P}=0.43)$, and 3 years $(2.0 \pm 0.1$ vs. $1.9 \pm 0.3 ; \mathrm{P}=0.90)$ were not significantly different, as shown in Figure 2. For the mitral valve, the mean gradients at baseline $(9.0 \pm 1.0 \mathrm{vs}$. $9.2 \pm 1.2 ; \mathrm{P}=0.73)$, 1 year $(5.5 \pm 0.4$ vs. $5.7 \pm 0.8 ; \mathrm{P}=0.52)$, and 3 years $(5.3 \pm 0.2$ vs. $5.5 \pm 0.7 ; \mathrm{P}=0.28)$ showed no statistically significant differences. Additionally, effective orifice areas at baseline $(1.2 \pm 0.2$ vs. $1.1 \pm 0.1 ; \mathrm{P}=0.50), 1$ year $(2.3 \pm 0.1$ vs. $2.2 \pm 0.2 ; \mathrm{P}=0.19)$, and 3 years $(2.4 \pm 0.2$ vs. $2.3 \pm 0.1 ; \mathrm{P}=0.26)$ had no statistical differences as shown in Figure 3. Hence, for both the aortic valve and the mitral valve, transthoracic Doppler echocardiography showed no statistically significant differences for mean gradient or effective orifice area at all-time points up to 3 years post-surgery.

\section{Hemocompatibility indexes}

A comparison of hemocompatibility indexes between the CL-V group and the SJM group is shown in Table 3. For hemoglobin, there were no significant differences at 6 months $(123.0 \pm 15.0$ vs. $137.3 \pm 17.8 ; \mathrm{P}=0.39)$ or 3 years $(120.0 \pm 13.2$ vs. $139.3 \pm 16.8 ; \mathrm{P}=0.49)$. For lactic dehydrogenase, there were no significant differences at 6 months $(215.6 \pm 74.5$ vs. $229.4 \pm 55.5 ; \mathrm{P}=0.83)$ or 3 years (211.6 \pm 76.5 vs. $239.2 \pm 54.5 ; \mathrm{P}=0.80$ ). Additionally, there were no significant differences in platelet count at 6 months (147.4 449.9 vs. $148.4 \pm 48.7 ; \mathrm{P}=0.98)$ or 3 years $(149.4 \pm 47.2$ vs. $149.4 \pm 43.1 ; \mathrm{P}=0.97)$.

\section{Discussion}

Our retrospective cohort study is the first observational study to evaluate the mid-term outcomes of the Chinesemade CL-V bileaflet mechanical heart valve after implantation in Chinese patients. The mid-term followup results of the Chinese-made CL-V bileaflet mechanical heart valves were similar to those of the St. Jude Medical heart valves, which showed stable hemodynamics and good blood compatibility.

An ideal artificial heart valve should have the following characteristics: (I) it should conform to blood hydrodynamic performance; (II) have good blood compatibility with low 
Table 3 Comparison of hemocompatibility indexes at 6 months and 3 years

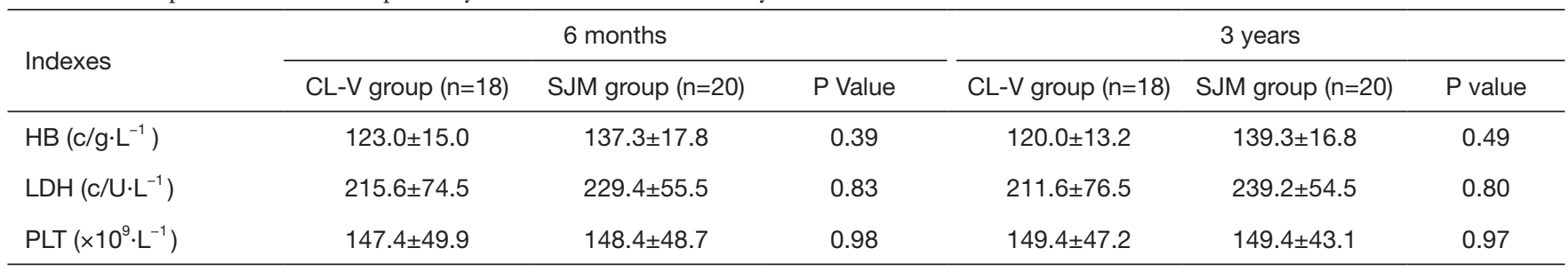

SJM, St. Jude mechanical heart valve; HB, hemoglobin; LDH, lactic dehydrogenase; PLT, platelet count.

noise, but no coagulation or hemolysis; (III) durability; (IV) ease of surgical implementation; (V) low cost and high availability. Since its first clinical application in 1977, the St. Jude Medical bileaflet prosthesis has been used worldwide due to its excellent hemodynamic characteristics and durability, and has come to represent the bileaflet prosthesis $(10,11)$. It was reported that the total number of mechanical heart valves decreased in terms of all prosthetic valves used in clinics, from $53 \%$ in 1996 to $32 \%$ in 2005 (12). The ability to design and develop Chinese-made bileaflet mechanical heart valves is still very poor.

The CL-V bileaflet mechanical heart valve was jointly developed by Changhai Hospital, the Second Military Medical University and Lanzhou LanFei Medical Instrument Co., Ltd. It was first used in clinical practice at our hospital in April 2004. The long-term followup results of the CL-V bileaflet mechanical heart valve after implantation in the human body were observed and analyzed, and were compared to 20 patients with the St. Jude Medical heart valve during the same period.

A comparison between the two groups in our study revealed no significant differences in demographic characteristics. The preoperative parameters were comparable for both groups of patients. All patients in both groups were successfully weaned from cardiopulmonary bypass $(\mathrm{CPB})$ with stable respiratory and circulatory systems, and survived the perioperative period. The smooth surface of the CL-V bileaflet and flexible transition between the open and closed positions were observed at follow-up. No significant differences in C/T, LAD, LVDD, LVEF and LVFS values at 1 year and 3 years post-surgery between the two groups were observed. The patients were able engage in manual labor at varying degrees, and achieved a good quality of life at follow-up. As the pressure difference across the valve can accurately reflect hemodynamic performance (13), our results demonstrated that the hemodynamic performance of the two valves was similar. No statistically significant differences in the mean transvalvular pressure gradient and effective orifice between the Chinesemade CL-V bileaflet mechanical heart valve and the St. Jude Medical heart valve were found. Furthermore, they are also similar to heart valves made in other countries $(14,15)$.

An intravascular hemolysis reaction is closely related to the transvalvular pressure gradient, prosthetic valve regurgitation and blood turbulent flow, which might indirectly reflect prosthetic valve hemodynamics $(16,17)$. Therefore, blood compatibility of prosthetic valves is another indicator for evaluating prosthetic valve performance. The results from our follow-up showed that there were no significant differences in hemocompatibility indexes between the CL-V bileaflet mechanical heart valve and the St. Jude Medical heart valve at 6 months and 3 years post-surgery. No hemolysis reaction or hemolytic anemia was noted. At present, prosthetic heart valve thromboembolism and anticoagulant-related bleeding are the two most important complications following mechanical prosthetic valve replacement (18), and are key to developing effective and safe prosthetic valves $(19,20)$. At recent follow-up, no thromboembolism or severe anticoagulantrelated bleeding was found with the same intensity as anticoagulation therapy (INR 1.5-2.5) for the two kinds of valves in this study. The results of the long-term efficacy of the Chinse-made CL-V bileaflet mechanical heart valve are still being observed.

According to comparisons between the two groups, the mid-term follow-up results of the CL-V bileaflet mechanical heart valve were similar to those of the St. Jude Medical heart valve, which demonstrated good mid-term clinical outcomes, stable hemodynamics and good blood compatibility. At recent follow-up, the cardiac function of the patients improved and there were no reports of valverelated complications, including valvular structural damage, paravalvular leakage, prosthetic valve endocarditis and thromboembolism, postoperative anticoagulation-related complications, and hemolytic anemia. Due to a limited number of cases in this study, further studies should be 
conducted using larger sample sizes of patients with the CL-V bileaflet mechanical heart valve, including its longterm outcomes.

\section{Study limitations}

The main limitation of this study was that it was a retrospective, single center study with a small sample size. We handle with the limitation through rigorously screened and excluded patients based on inclusion and exclusion criteria.There was also a lack of postoperative complications to evaluate the safety of the CL-V bileaflet mechanical heart valve. Moreover, the endpoints lacked an assessment of postoperative complications. Further studies with a larger sample size, a longer follow-up or a randomized prospective controlled trial are needed to evaluate the Chinese-made CL-V bileaflet mechanical heart valve.

\section{Conclusions}

The mid-term follow-up results of the Chinese-made CL-V bileaflet mechanical heart valve was similar to those of the St. Jude Medical heart valve, which showed stable hemodynamics and good blood compatibility. CL-V bileaflet mechanical heart valves can be a substitute for St. Jude Medical heart valves, and can be widely used in cardiac surgery.

\section{Acknowledgments}

We thank all the authors for their wonderful collaboration.

Funding: This work was supported by the National Natural Science Foundation of China (Grant No. 51966010); Retrospective observational study on Chinese-made CL-V full-carbon bileaflet mechanical heart valve (Grant (19) 0564); Natural Science Foundation of Gansu Province (20JR10RA689).

\section{Footnote}

Reporting Checklist: The authors have completed the STROBE reporting checklist. Available at http://dx.doi. org/10.21037/jtd-20-2644

Data Sharing Statement: Available at http://dx.doi. org/10.21037/jtd-20-2644

Conflicts of Interest: All authors have completed the ICMJE uniform disclosure form (available at http://dx.doi. org/10.21037/jtd-20-2644). The authors have no conflicts of interest to declare.

Ethical Statement: The authors are accountable for all aspects of the work in ensuring that questions related to the accuracy or integrity of any part of the work are appropriately investigated and resolved. The study was approved by the ethics committee of the First Hospital of Lanzhou University (LDYYLL2019-230), and the study was registered with the Chinese Clinical Trial Registry, registration number ChiCTR2000034158. Informed consent was obtained from all individual participants included in the study. All procedures performed in this study involving human participants were in accordance with the Declaration of Helsinki (as revised in 2013).

Open Access Statement: This is an Open Access article distributed in accordance with the Creative Commons Attribution-NonCommercial-NoDerivs 4.0 International License (CC BY-NC-ND 4.0), which permits the noncommercial replication and distribution of the article with the strict proviso that no changes or edits are made and the original work is properly cited (including links to both the formal publication through the relevant DOI and the license). See: https://creativecommons.org/licenses/by-nc-nd/4.0/.

\section{References}

1. Domenech B, Pomar JL, Prat-González S, et al. Valvular Heart Disease Epidemics. J Heart Valve Dis 2016;25:1-7.

2. upino PG, Goon AK, Borer JS. The Epidemiology of Valvular Heart Diseases: Incidence Growing. Circulation 2010;122:abstr 10314.

3. Lipton MJ, Coulden R. Valvular heart disease. Radiol Clin North Am 1999;37:319-39, v-vi.

4. Iung B, Vahanian A. Epidemiology of acquired valvular heart disease. Can J Cardiol 2014;30:962-70.

5. Zhang Y, Ma L. Identification of key genes and pathways in calcific aortic valve disease by bioinformatics analysis. J Thorac Dis 2019;11:5417-26.

6. Xu JB, Xu ZY, Zhang BR, et al. Home-made C-L pugesturt tilting disc: a 12 years follow-up of 538 cases. Academic Journal of Second Military Medical University 2007;28:1021-3.

7. Bark DL Jr, Vahabi H, Bui H, et al. Hemodynamic Performance and Thrombogenic Properties of a Superhydrophobic Bileaflet Mechanical Heart Valve. Ann 
Biomed Eng 2017;45:452-63.

8. Xu R, Rahnavardi M, Pitman B, et al. On-X versus St Jude Medical Regent mechanical aortic valve prostheses: early haemodynamics. Open Heart 2017;4:e000539.

9. Zhu X, Luo Y, Zhang E, et al. Ten-year experience of tricuspid valve replacement with the St. Jude medical valve. Sci Rep 2018;8:16654.

10. Bernet FH, Baykut D, Grize L, et al. Single-center outcome analysis of 1,161 patients with St. Jude medical and ATS open pivot mechanical heart valves. J Heart Valve Dis 2007; 16:151-8.

11. Cianciulli TF, Lax JA, Beck MA, et al. Valve replacement with bileaflet HP-Biplus mechanical prostheses versus standard prostheses: long-term results. J Heart Valve Dis 2008;17:206-15.

12. Tossios P, Reber D, Oustria M, et al. Single-center experience with the On-X prosthetic heart valve between 1996 and 2005. J Heart Valve Dis 2007;16:551-7.

13. Bonow RO, Carabello BA, Chatterjee K, et al. 2008 focused update incorporated into the ACC/AHA 2006 guidelines for the management of patients with valvular heart disease: a report of the American College of Cardiology/American Heart Association Task Force on Practice Guidelines (Writing Committee to revise the 1998 guidelines for the management of patients with valvular heart disease). Endorsed by the Society of Cardiovascular Anesthesiologists, Society for Cardiovascular Angiography

Cite this article as: Qi L, Liu S, Qiang Y, Zhao H, Liu R, Cheng D, Song B, Guan Q. Clinical mid-term outcomes of the Chinese-made CL-V bileaflet mechanical heart valve in Chinese patients. J Thorac Dis 2021;13(1):10-17. doi: 10.21037/jtd-202644 and Interventions, and Society of Thoracic Surgeons. J Am Coll Cardiol 2008;52:e1-e142.

14. Rosenhek R, Binder T, Maurer G, et al. Normal values for Doppler echocardiographic assessment of heart valve prostheses. J Am Soc Echocardiogr 2003;16:1116-27.

15. Remadi J P, Bizouarn P, Baron O, et al. Mitral valve replacement with the St. Jude Medical prosthesis: a 15year follow-up. Ann Thorac Surg 1998;66:762-7.

16. Josa M, Castella M, Pare C, et al. Hemolysis in mechanical bileaflet prostheses: experience with the Bicarbon valve. Ann Thorac Surg 2006;81:1291-6.

17. Mecozzi G, Milano A D, De Carlo M, et al. Intravascular hemolysis in patients with new-generation prosthetic heart valves: a prospective study. J Thorac Cardiovasc Surg 2002;123:550-6.

18. Fu B, Chen H, Dong L. Antithrombotic and thrombolytic therapy for valvular disease: can this guideline apply to Chinese? Chest 2013;143:1513-4.

19. Misawa Y, Saito T, Konishi H, et al. Clinical experience with the Bicarbon heart valve prosthesis. J Cardiothorac Surg 2007;2:8.

20. Le Tourneau T, Lim V, Inamo J, et al. Achieved anticoagulation vs prosthesis selection for mitral mechanical valve replacement: a population-based outcome study. Chest 2009;136:1503-13.

(English Language Editor: C. Belazar-Maseh) 